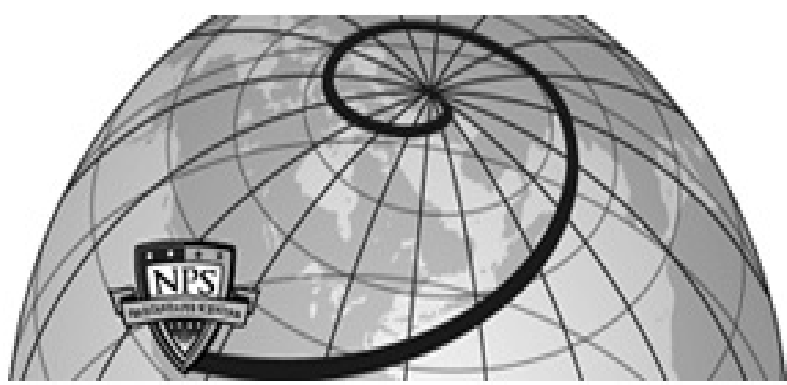

Calhoun: The NPS Institutional Archive DSpace Repository

1951

\title{
Transport parameters for thermal neutrons in water.
}

Sisk, Francis Joseph

Monterey, California: U.S. Naval Postgraduate School

https://hdl.handle.net/10945/14738

Downloaded from NPS Archive: Calhoun

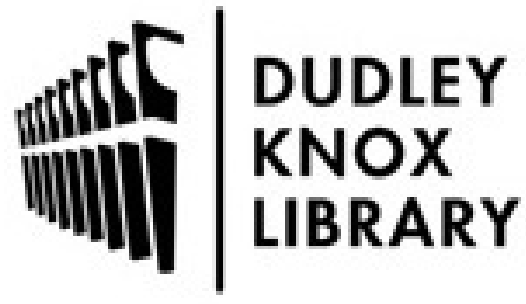

http://www.nps.edu/library
Calhoun is the Naval Postgraduate School's public access digital repository for research materials and institutional publications created by the NPS community. Calhoun is named for Professor of Mathematics Guy K. Calhoun, NPS's first appointed -- and published -- scholarly author.

Dudley Knox Library / Naval Postgraduate School 411 Dyer Road / 1 University Circle Monterey, California USA 93943 


\title{
TRANSPORT PARAMETERS FOR THERMAL NEUTRONS IN WATER
}

\author{
BY \\ FRANCIS JOSEPH SISK.
}


Library

ש. 8. Naval Peatgraduat School Jimapolis, Md. 

TRANSPORT PARAMETERS FOR THERMAL NEUTRONS IN WATER

\author{
by \\ Francis Joseph Sisk \\ Lieutenant, United States Navy
}

Submitted in partial fulfillment

of the requirements

for the degree of

MASTER OF SCIENCE

United States Naval Postgraduate School

Annapolis, Maryland

1950 
This work is accepted as fulfilling the thesis requirements for the degree of MAST LR OF SCINCE

from the

United States Naval Postgraduate School 


\section{TABLE OF CONTENTS}

$\begin{array}{llr}\text { Chapter I. } & \text { Page } \\ & \text { I. Introduction and Summary } & 1 \\ & \text { 2. Summary } & 1 \\ \text { Chapter II. } & \text { Transport Theory and Methods of Analysis } & 5 \\ \text { Chapter III. } & \text { Experimental Procedure } & 22 \\ \text { Bibliography } & & 32 \\ \text { Appendix I } & & 33 \\ \text { Appendix II } & \end{array}$



$\underline{\text { Page }}$

1. Velocity Diagran for Two-Body Collision 26

2. Integration for Plane Kernel - Transport Equations $\quad 27$

3. Foil kount and Cadmium Plane Assembly 28

4. Thermal Neutron Flux in Water Near a Black Plane 29

5. One-Dimensional Thermal Neutron Flux in Water Near 30 a Black Boundary

6. Diametral Flux Distribution

31 
CHAPTER I

\section{INTRODUCTION AND SESIIARY}

1. Introduction

The steady state spatial distribution of neutron fluxes in matter is a problem of prime importance to the designer of chain reacting piles, and has been the subject of very much theoretical and experimental work. The case in which the neutron energy is large compared to that of the nuclei constituting the mediun (the "slowing down" case) is of first importance and is rather extensively investigated. The alternate case in which the neutrons are in energy equilibrium with the medium is of lesser importance and conparatively little experimental work has been done, although the fundamental theory is well in hand. The cross-sections for neutron scattering and absorption appropriate to the first case are the "ordinary" energy-dependent cross-sections (or simple functions of these) which have been so extensively investigated by various neutron spectrometry groups. The cross-sections appropriate to the second case are more vaguely defined. Perhaps as adequate a definition of these parameters as any is the "ad hoc" one - those numbers which, when inserted in the mathematical theory of particle transport yield proper solutions for the neutron flux. It can be demonstrated that these numbers are not in general the average values of the energy dependent cross sections weighted by the appropriate energy distribution function for the ambient temperature.

The "ad hoc" definition suggests a means of experimentally measuring the numbers. One requires a medium - of simple geometry so that the transport problem is soluble - in which a flux of thermal equilibrium noutrons 
is maintained and in which the flux can be measured at various points with neutron detectors. Since two parameters are to be determined, two independent solutions for the flux must be available - but this is easily provided for by considering (1) a region "remote" from discontinuities in which the solution is asymptotic and (2) a region adjacent to a discontinuity - say a "black" boundary. One measures the flux at a sufficient number of points in each region to define the spatial functions. The transport problem is solved many times for various values of the parameters, and if fortune is good one pair of values will be found to yield solutions which fit the experimental functions in both regions.

To particularize - the chosen medium is normal water, the geometry one-dimensional consisting of a tank many mean free paths in its dimensions, traversed by a uniform thermal neutron current arriving at one face and absorbed by a layer of cadmium at the opposite face. The neutron flux is measured at points along a line normal to the cadmium facing and extending from the facing well into region (1).

The one-dimensional transport problem is integrable in closed form for region (1). This solution is matched to the experimental data, yielding a characteristic equation in say the total cross-section and the scattering cross-section - thus the scattering section may be eliminated. The transport problem is then integrated numerically in region (2) for various values of total cross section and that value selected which yields best fit with experimental data. Unfortunately, this straight-forward procedure could not be carried out. It was found that numerical integration produced a function which fit the experimental data astonishingly well, but that the values of the parameter which yielded this good fit were variable with 
respect to the integrating procedure. Further, it was found that each improvement in the integrating plan led monotonously to a reduction in the total cross section, indicating that the process of improving the operation was not near convergence. The rapid increase in computing labor made it necessary to abandon this process before a firm solution was obtained.

The Wiener-Hopf method, treated by Frankel and Goldberg (3), offers a general solution to the one-dimensional transport problem in a single semi-infinite medium. However, it is available only as an exceedingly difficult double numerical quadrature which could not be handled for lack of computing facility. A solution is given for the flux at the black boundary only, requiring only one numerical quadrature. By equating this solution in terms of the parameters to the experimental value of the "contact" flux, unique values are obtained for the parameters. These values extrapolate the trend of the numerical solutions, and are in that sense to be regarded as supported by the numerical method.

The convergence on the Wiener-Hopf solution may be illustrated by the successive values for the macroscopic "total" cross section. They are: Numerical $-2.9 \mathrm{~cm}^{-1}, 2.5 \mathrm{~cm}^{-1}$ and $2.3 \mathrm{~cm}^{-1}$; Wiener-Hopf $-2.10 \mathrm{~cm}^{-1}$. 
2. Summary

Findings may be tabulated as follows:

Parameter - Water at $24^{\circ} \mathrm{C}$. Values

\begin{tabular}{ll}
\hline Diffusion Length & $2.67 \pm .02 \mathrm{~cm}$. \\
\hline Transport Mean Free Path & $0.48 \pm .01 \mathrm{~cm}$. \\
\hline Absorption Nean Path (Equivalent) & $44.2 \pm 1.3 \mathrm{~cm}$. \\
\hline $\begin{array}{l}\text { Microscopic Transport Cross Section } \\
\text { Sicroscopic Absorption Cross } \\
\text { Section (Equivalent) }\end{array}$ & $62.4 \pm 1.5 \mathrm{barns} / \mathrm{mol}$. \\
\hline $\begin{array}{l}\text { Ratio - Boundary Flux } \div \text { Asymptotic } \\
\text { (Exponential) Flux }\end{array}$ & $0.68 \pm 0.01 \mathrm{barns} / \mathrm{mol}$. \\
\hline $\begin{array}{l}\text { Ratio - Boundary Flux } \div \text { Asymptotic } \\
\text { (Hyperbolic) Flux }\end{array}$ & 0.186 \\
\hline $\begin{array}{l}\text { Extrapolation Distance - Xo } \\
\text { Ratio Extrapolation Distance/Total } \\
\text { Mean Path }\end{array}$ & 0.87 \\
\hline
\end{tabular}

Comparison of the cross-section with the energy-dependent values for protons in water by Bacher(1) and Melkonian (7) indicate an "average" neutron energy for transport of perhaps 0.05 electron volts in contrast with the Yaxwell-Bolt zmann mode of $0.025 \mathrm{e} . \mathrm{v}$. This is not surprising since it is expected that transport is largely effected by the high energy neutrons which have longer free paths. In addition it must be remembered that the neutron flux is weighted by a velocity term so that the average flux corresponds to a higher energy than does the average neutron, and transport weights the cross-section by the flux rather than by the neutron density. The $1 / v$ absorption in hydrogen provides a preferential sink for low energy neutrons, thus the thermal distribution is displaced from the Maxwell-Boltamann toward higher energy. 
CHAPTER II

\section{TRANSPORT THEORY AND METHODS OF ANALYSIS}

To indicate the assumptions involved and to portray the physical model which was analyzed, it may be well to review the fundamental theory of neutron transport. It must be emphasized that the model of neutron transport for neutrons in energy equilibrium with the medium, at energies well below the atomic binding, differs radically from that for energetic neutrons $\left(E_{n}>1 \mathrm{ev}\right)$ in media at normal temperature.

In the latter case the energy transfer in neutron-nucleus interactions is uniformly from neutron to nucleus, the nuclei interact as free particles, and since the atomic vibrational energy is comparatively small, the nuclei may be regarded as initially at rest. None of these statements hold for the former case.

In the present problem, by definition, the net average energy transfer between neutrons and medium is very small and due solely to the preferential absorption of the slower neutrons. The protons are bound in the molecule with energy larger than the neutron energy and the nuclei cannot interact as free particles. The thermal motion of the atoms and of the systems in which they are bound is not negligible, and so affects not only the mechanics of the collisions but also the frequency of occurrence since the collision probability is a function of the flux of nuclei as well as of neutrons.

Inelastic interation of neutrons with rotational and vibrational levels of the water molecule are probably present. The rotational levels are quite numerous and begin at wave number $23.8 \mathrm{~cm}^{-1}$, (about .003 electron volts) according to Wu (9).

Quantitive discussion of the scattering mechanism in a system as 
complex as this is not in the scope of this paper. However to avoid misinterpretation of the results, it is necessary. to consider at least the question of whether the scattering is symetric, that is whether all values of the angle of deflection of the neutron in the Laboratory System are equally probable, when the neutrons are in energy equilibrium with the medium.

Referring to Fig. (1), $\bar{v}_{0}$ and $\bar{v}$ are the velocities of reutron and molecule respectively before collision, in the laboratory system, and $\boldsymbol{\omega}$ the angle between then. To transfer to the center of mass system a vector velocity $\bar{V}$ is added of such magnitude and direction as to reduce the total momentum to zero. Using unity as neutron mass and $M$ as molecule mass we have:

$$
v_{0}+V_{x}+M\left(v \cos \omega+V_{x}\right)=0
$$

and

$$
V_{y}+M\left(v \sin \omega+V_{y}\right)=0
$$

thus

$$
v_{x}=-\frac{M}{M+1} v \cos \omega-\frac{1}{M+1} v_{0}
$$

and

$$
V_{y}=-\frac{M}{M+1} v \sin \omega
$$

where $V_{x}$ and $V_{y}$ are the scalar components of $\bar{V}$.

The transformation vector is thus seen to resolve into two vectors, one of magnitude $\frac{M}{M+1} v$ and opposite to $\bar{v}$ in direction, and one of ragnitude $\frac{1}{\mathrm{M}+1} v_{0}$ and opposite to $\bar{v}_{0}$ in direction. The vectors $\bar{v}_{0}^{\prime}$ and $\bar{v}^{\prime}$ are the velocities before collision in c... systel and $\bar{v}_{0}^{\prime \prime}$ and $\overline{\boldsymbol{v}}^{\prime \prime}$ are the C.Nio velocities after collision. If the scatterin s sherically symmetric in C. Whe system, all values of $\cos \psi$, the deflection angle, are

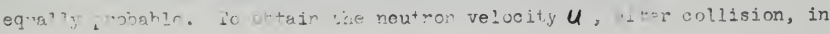


Laboratory system, we add $(-\bar{v})$ vectorially to $\bar{\gamma}_{0}^{\prime \prime}$. The probability of $\omega$ is sin $\omega$, and is therefore symmetric, so on the average the contribution of $\frac{M}{M+1} \bar{v}$ involves no preferred direction. However, the contribution of $\frac{1}{M+1} \bar{v}_{0}$ inserts the direction of the incoming neutron as a component of $u$. Thus the scattered neutrons will emerge from collisions preferentially in the direction of their pre-collision motion with a weight increasing with neutron energy.

The preferentially forward scattering, as indicated, correlates rather poorly the direction of each path with the preceding one, less well with the next but one preceding, and after a large number of intervening scatterings the correlation vanishes. One may attempt to obtain the number of neutrons passing through a small volume increment $(d V)$ by summing up those neutrons scattered in all other volume increments $\left(d \gamma^{\prime}\right)$ in the space which reach the first increment in free flight. However, since the number of scattered neutrons leaving $d V^{\prime}$ in the direction of $d \gamma$ depends on the average direction of the neutrons entering $d V^{\prime}$, this average direction must be found by another summing up of previously scattered neutrons and so on. The mathematical analysis of such a situation yields infinite order multiple integrals, so simplification is necessary. The difficulty does not occur if the scattering is isotropic, so an obvious suggestion might be to approximate the system with a model in which the scattering is isotropic.

If one considers a neutron scattered isotropically at a point in a nonabsorbing medium and asks for the probability of finding it at any point in the space after the lapse of a long time, it is clear that the resulting probability field is symmetric about the initial point. Analogously, one considers a neutron with initial motion $\bar{\gamma}_{0}$ scattered at a point anisotropically in the Lab system but isotropically in C.M. system. 
The probability of finding the once scattered neutron at position $\bar{r}$ is then symmetric about the moving center of mass whose velocity is $\frac{1}{M+1} \bar{v}_{0}$. However, the probability of finding the second scattered neutron at $\bar{r}$ is only azimuthally symmetric since each spherical shell of second scattered neutron probability is immediately again retarded by the factor $\frac{1}{M+1}$, corresponding to the motion of the center of mass of the second scattering system. Thus the second scattered neutrons which have made long flight after first scattering will be found on the average farther along in the direction of $\bar{v}_{0}$ than those which make short flights. In spite of the forbidding complexity of the picture some information can be obtained about the probability distribution after a large number of collisions. The path lengths $\boldsymbol{l}$, the azimuthal scattering angles $\boldsymbol{l}$, and the cosines of the polar scattering angles $\mu$, are every one statistically independent, and may be averaged independently. The centroid of the distribution is obviously found in the direction of $\bar{v}_{0}$ and may be located by first averaging over the azimuthal angles starting with the last flight. One obtains $\left(l_{1} \mu_{1}+l_{2} \mu_{2} \mu_{1}+\ldots l_{n} \prod_{1}^{n} \mu_{n}+\ldots\right)$. Averaging over $l_{n}$ and $\mu_{n}$ one has

$\bar{l}=\iint \mu_{1} P_{\left(\mu_{1}\right)} \ell_{1} P_{\left(l_{1}\right)} d \mu_{1} d l_{1}+\iiint \mu_{1} P_{\left(\mu_{1}\right)} \mu_{2} P_{\left(\mu_{2}\right)} l_{2} P\left(l_{2}\right) d \mu_{1} d \mu_{2} d l_{2}+\ldots$ The required first moment is then $\lambda_{s}\left(\bar{\mu}+\bar{\mu}^{2}+\bar{\mu}^{3}+\ldots \bar{\mu}^{n}+\ldots\right)$ which is the expansion of $\frac{\lambda_{s} \bar{\mu}}{1-\bar{\mu}}$. One concludes then that the average position, after very many scatterings, of a neutron projected from a point in a medium is at a distance $\lambda_{s}\left(1+\frac{\bar{\mu}}{1-\bar{\mu}}\right)$ or $\frac{\lambda_{s}}{1-\bar{\mu}} \quad$ from the initial point. This parameter is called the transport mean free path. The higher moments of the probability function are not available in general. 
F. Joliot (5) has computed the probability function itself for the $\mathrm{n}^{\text {th }}$ isotropic scattering in a quite complicated series of Hermite polynomials.

One may now reasonably postulate that the scattering mechanism in an anistropically scattering medium may be rather well reproduced by an isotropic model, provided that the scattering length be weighted by the factor $\frac{1}{1-\bar{\mu}}$. This ignores the defect that the higher moments are not properly scaled.

It must be emphasized that capture has not been considered and that the model is not correct for large capture. It then remains to be demonstrated that the model is satisfactory if capture is small but not negligible.

The simplified transport model now may be more clearly defined. It consists of a three dimensional array of fixed lattice points at which the neutrons scatter isotropically, or are absorbed.

The scattering length in the model is the transport free path in the real medium and is in general not known. The absorption free path in the model is not in general the same as the absorption length in the medium but is probably nearly the same if absorption is small. A more useful pair of parameters are the inverses of the free paths - the macroscopic cross sections - defined as the probability per centimeter of path of the defined event. To prove the relation:

$$
\begin{aligned}
-d I & =I \Sigma d l \\
I & =I_{0} \varepsilon^{-\Sigma l}
\end{aligned} \quad \text { Where } I \text { is the beam intensity }
$$

Let $I_{0}=1, \quad I=\epsilon^{-\sum l}$ The mean value of $l$ is:

$$
\lambda=\frac{\int_{0}^{\infty} l e^{-\Sigma l} d l}{\int_{0}^{\infty} \epsilon^{-\Sigma l} d l}=\frac{1}{\Sigma}
$$


. 
A macroscopic absorption cross-sectior is similarly defined.

The following notation is to be used:

n - Neutron density, $\mathrm{cm}^{-3}$

$\varphi$ - Neutron scalar flux density, called simply "flux, " $\mathrm{cm}^{-2} \mathrm{sec}^{-1}$

$\Sigma_{t}$ - Mean macroscopic transport cross section, $\mathrm{cm}^{-1}$

$\Sigma_{a}$ - Nean macroscopic absorption cross section $\mathrm{cm}^{-1}$

$\sum$ - Nean total cross section, $\mathrm{cm}^{-1}$

$N$ - Lattice point density of the medium, $\mathrm{cm}^{-3}$

$\sigma \quad$ - Nicroscopic total cross section, $\mathrm{cm}^{2}$

$x$ - Distance, om.

$Z$ - Distance in mean paths

$\bar{r} \quad$ - Position vector

One asks for the number of neutrons scattered in incremental volume at position $\bar{r}$ and crossing unit normal area at $\bar{r}^{\prime}$ per second. The total scattered at $\bar{r}$ is $\varphi(\bar{r}) \Sigma_{t} d V$ - all cross sections being independent of $\bar{r}$ in the homogeneous medium - and of these $\frac{1}{4 \pi} d \omega$ leave $\bar{r}$ in $d \omega$ about solid angle $\omega$. Since the area subtended at $\bar{r}^{\prime}$ by dw at $\bar{r}$ is $\left(\bar{r}^{\prime}-\bar{r}\right)^{2} d \omega$, a division by this factor is indicated, and since the beam in any $d \omega$ is attenuated by absorption and re-scattering as

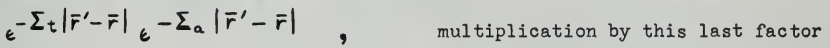
is required. The required number is then

$$
\frac{\varphi_{(\bar{r})} \Sigma_{t} \epsilon-\Sigma\left|\bar{r}^{\prime}-\bar{r}\right| d \gamma}{4 \pi\left(\bar{r}^{\prime}-\bar{r}\right)^{2}}
$$

The neutron flux at $\bar{r}^{\prime}$ is then obtained by summing up the neutrons scattered at all $\overline{\boldsymbol{r}}$ and crosing unit area at $\overline{\boldsymbol{r}}^{\prime}$. Thus

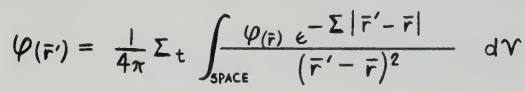


In the proklem at hand, the medium is considered to be a semi-infinite slab - infinite in horizontal extent and downward, its upper face bounded by a plane from which no neutrons return, and having a finite neutron current passing upward through it. The coordinate $x$ is measured from the "black" plane and normal to it. In this system the flux is a function of $\boldsymbol{x}$ alone.

One requires the number of neutrons scattered in an incremental slab $d x$ at $x$ and passing in free flight through unit area about a point at $x^{\prime}$. Referring to Fig. 2, this quantity called $K_{\left(\left|x^{\prime}-x\right|\right)} d x$ is found as follows:

$$
\begin{gathered}
K d x=\frac{\varphi(x) \Sigma_{t}}{4 \pi} \int_{r=0}^{\infty} \frac{e^{-\Sigma p}}{\rho^{2}}(2 \pi r d r d x) \\
r^{2}+\left(x^{\prime}-x\right)^{2}=\rho^{2} \\
r d r=\rho d \rho \\
K=\frac{\varphi(x) \Sigma_{t}}{2} \int_{p=\left|x^{\prime}-x\right|}^{\infty} \frac{\varepsilon^{-\Sigma p}}{\rho} d p \\
K=\frac{\varphi(x) \Sigma_{t}}{2} \int_{\Sigma\left|x^{\prime}-x\right|}^{\infty} \frac{e^{-\Sigma p}}{\Sigma p} d(\Sigma p)
\end{gathered}
$$


The integral is terred $E_{1}\left(\Sigma\left|x^{\prime}-x\right|\right)$, the exponential integral, and is identical with the function $-E_{t}(-x)$ as tabulated by Jahnke \& Ehmde and others.

The flux at $x^{\prime}$ is obtained by summing the contributions of all increments $d x$ at $x$ as follows:

$$
\varphi\left(x^{\prime}\right)=\frac{\Sigma_{t}}{2} \int_{\text {space }} \varphi(x) E_{1}\left(\Sigma\left|x^{\prime}-x\right|\right) d x
$$

This linear integral equation is perhaps more conveniently handled in mean free path dimensions as follows:

$$
\varphi_{\left(z^{\prime}\right)}=\frac{\Sigma_{t}}{2 \Sigma} \int \varphi(z) E_{1\left(\left|z^{\prime}-z\right|\right)} d z
$$

For the configuration space described, the limits of integration are zero and plus infinity. However as $-Z \geq-15$ the contribution to the integral between negative infinity and zero becomes negligibly small, and the limits may be taken, with vanishing error, over all values of $z$. In this asymptotic form the equation has a simple hyperbolic solution.

The usual method of treatment, to indicate the form of the asymptotic solution (as in Frankel \& Nelson (4)), involves transforming the origin to write:

$$
\varphi\left(z^{\prime}\right)=\frac{\Sigma_{t}}{2 \Sigma} \int_{-\infty}^{\infty} \varphi_{\left(z^{\prime}+z\right)} E_{1(z)} d z
$$

and expand $\varphi\left(z^{\prime}+z\right)$ in Taylor's series about $z^{\prime}$ :

$$
\begin{aligned}
& \varphi\left(z^{\prime}\right)=\frac{\Sigma_{t}}{2 \Sigma} \int_{-\infty}^{\infty} E_{1(|z|)}\left[\varphi\left(z^{\prime}\right)+z \varphi_{\left(z^{\prime}\right)}^{\prime}+\frac{z^{2}}{2 !} \varphi^{\prime \prime}\left(z^{\prime}\right)+\cdots\right] d z \\
& \text { Now } m_{m}=\int_{-\infty}^{\infty} z^{m} E_{1}(|z|) d z \quad \text { is the } m^{\text {th }} \text { mont of the kernel }
\end{aligned}
$$


about its origin, and this vanishes by symmetry for $m$ odd. One has then

$$
\varphi_{\left(z^{\prime}\right)}=\frac{\Sigma_{t}}{2 \Sigma}\left[m_{0} \varphi_{\left(z^{\prime}\right)}+\frac{m_{2}}{2} \varphi^{\prime \prime}\left(z^{\prime}\right)\right]
$$

neglecting terms above the third order. Rearranging, one has the onedimensional differential equation of elementary diffusion theory - the Helmholtz equation:

$$
\varphi^{\prime \prime}+\left(\frac{2 m_{0}}{m_{2}}-\frac{4 \Sigma}{m_{2} \Sigma_{t}}\right) \varphi=0
$$

for which the solutions are hyperbolic if $m_{0}<\frac{2 \Sigma}{\Sigma_{t}}$, circular if $m_{0}>\frac{2 \Sigma}{\Sigma_{t}}$, and linear if $m_{0}=\frac{2 \Sigma}{\Sigma_{t}}{ }^{\Sigma_{t}}$. Now $m_{0}=\int_{-\infty}^{\infty} E_{1(|z|)} d z=2$, and if absorption is present, then $\Sigma>\Sigma_{t}$, so $m_{0}=2<\frac{2 \Sigma}{\Sigma_{t}}$. Thus we expect hyperbolic solutions, so a trial function $\varphi_{(z)}=\epsilon^{-\not z}$ is substituted in the integral equation, and the integration performed at the point $z^{\prime}=0$

$$
\begin{aligned}
& 1=\frac{\Sigma_{t}}{2 \Sigma} \int_{-\infty}^{\infty} \epsilon^{-\ell l z} E_{1}\left(\left|z^{\prime}-z\right|\right) d z \\
& =\frac{\Sigma_{t}}{2 \Sigma}\left\{\int_{-\infty}^{0} \epsilon^{-\not z} E_{1(-z)} d z+\int_{0}^{\infty} e^{-\not z} E_{1}(z) d z\right\} \\
& =\frac{\Sigma t}{2 \Sigma}\left\{\int_{0}^{\infty} \epsilon^{+d z} E_{1}(z) d z+\int_{0}^{\infty} \epsilon^{-x z} E_{1}(z) d z\right\} \\
& =\frac{\Sigma t}{\Sigma} \int_{0}^{\infty} \cosh (\not z) E_{1}(z) d z
\end{aligned}
$$

by parts after putting $E_{1}(z)$ in integral form.

$$
1=\frac{\Sigma_{t}}{\Sigma} \int_{0}^{\infty} \cosh (\mathscr{R} z) d z \int_{z}^{\infty} \frac{e^{-y}}{y} d y
$$




$$
1=\frac{\Sigma_{t}}{\Sigma}\left[\frac{1}{\mathscr{d}} \sinh (\mathscr{H} z) E_{1}(z)\right]_{0}^{\infty}+\frac{\Sigma_{t}}{x \Sigma} \int_{0}^{\infty} \sinh (\mathscr{P} z) \frac{\varepsilon^{-z}}{z} d z
$$

The first integral vanishes for $\boldsymbol{R}<1$ since at upper limit $E_{1}(z)$ is of order $\varepsilon^{-Z}$ and at lower limit $\frac{\sinh R \mathcal{Z}}{\mathscr{\ell}}$ goes as $z$ and the singularity of $E_{1}(z)$ is logarithmic. Writing the second integral in exponential form

$$
1=\frac{\Sigma_{t}}{2 R \Sigma} \int_{0}^{\infty} \frac{e^{-Z(1-x)}-\varepsilon^{-z(1+x)}}{Z} d z
$$

and separating the range of integration into intervals:

$$
1=\frac{\Sigma_{t}}{2 x \Sigma}\left\{\int_{0}^{1 / 1-x} \frac{\varepsilon^{-z(1-x)}}{z} d z-\int_{0}^{1 / 1+x} \frac{\varepsilon^{-z(1+x)}}{z} d z+\int_{\frac{1}{1-x}}^{\frac{-z(1-x)}{z}} d z-\int_{\frac{1}{1+x}}^{\frac{\varepsilon^{-z}(1+x)}{z}} d z\right\} .
$$

the third and fourth integrals are seen to be each $\mathrm{E}_{1}(1)$ and cancel, thus

$$
1=\frac{\Sigma_{t}}{2 \not R} \ln \frac{1+\not R}{1-\not H}
$$

or $\frac{\Sigma_{t}}{\Sigma}=\frac{\not}{\frac{1}{2} \ln \frac{1+\not x}{1-\not h}}=\frac{\not}{\tanh ^{-1} x}$

expanding in power series

$$
\frac{\Sigma}{\Sigma t}=1+\frac{x^{2}}{3}+\frac{x^{4}}{5}+\ldots+\frac{x^{2 n}}{2 n+1}
$$

Inverting the series in $\mathbb{R}^{2}$ we obtain

$$
R^{2}=3 \frac{\Sigma_{a}}{\Sigma_{t}}-\frac{9}{5}\left(\frac{\Sigma_{a}}{\Sigma_{t}}\right)^{2}-1.851\left(\frac{\Sigma_{a}}{\Sigma_{t}}\right)^{3}+\cdots
$$

Note that $\epsilon^{-\not Z Z}$ is an eigen function in mean free path measure and the function in cr: measure is $\epsilon^{-k x}$ where $k=\not \ell \Sigma$.

14 
Given an experimertal value of $\mathbf{k}$ (which is simply the logarithmic slope of the flux distribution plotted against $\boldsymbol{x}$ ) one may choose a value of $\Sigma$, compute $\mathscr{R}$ by $\mathscr{H}=\frac{k}{\Sigma}$, and using the series in $\mathbb{H}^{2}$ (or any conveniently obtained solution) compute $\Sigma t / \Sigma$. Returning to the integral equation

$$
\varphi(z)=\frac{\Sigma t}{2 \Sigma} \int_{0}^{\infty} \varphi\left(z^{\prime}\right) E_{\left(\left|z^{\prime}-z\right|\right)} d z^{\prime}
$$

one may insert the computed value of $\Sigma t / \Sigma$. It is now required to compute the eigenfunction $\varphi_{(z)}$ corresponding to the selected value of $\Sigma$. A choice of method is available.

LeCaine $(6)$ has treated the problem by a variational method, presenting tabulated values for the flux normalized to unit flux at the black plane for various values of $\Sigma_{a} / \Sigma_{t}$. A rather rough solution for the parameters is available by using these results, and indicates that $\Sigma$ lies between $2.0 \mathrm{~cm}^{-1}$ and $2.4 \mathrm{~cm}^{-1}$.

It was then decided to attempt solution by a direct numerical method, which was carried out to completion. In theory the method goes as follows: Given the integral equation as before

$$
\varphi(z)=\frac{\Sigma t}{2 \Sigma} \int_{0}^{\infty} \varphi\left(z^{\prime}\right) E_{1}\left(\left|z^{\prime}-z\right|\right) d z^{\prime}
$$

The integral is replaced by a Riemann sum equation

$$
\varphi\left(z_{i}\right)=\frac{\Sigma_{t}}{2 \Sigma} \Delta z \sum_{r_{n}} \varphi_{\left(z_{n}\right)} E_{1}\left(\left|z_{i}-z_{n}\right|\right)
$$

which may be put in matrix notation 


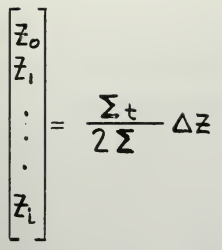

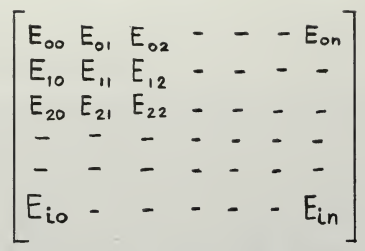$$
\left[\begin{array}{l}
z_{0} \\
z_{1} \\
z_{2} \\
- \\
- \\
z_{n}
\end{array}\right]
$$

where $E_{\text {in }}$ is the absolute value of the exponential integral of argument $(i-n) \Delta Z$. At the argument $i=n$ the exponential integral is singular, so a mean value was computed for this range. One requires:

$$
\begin{aligned}
& \frac{2}{\Delta z} \int_{0}^{\frac{\Delta z}{2}} E_{1}(z) d z=\frac{2}{\Delta z} \int_{0}^{\frac{\Delta z}{2}} d z \int_{1}^{\infty} \frac{e^{-y^{z}}}{y} d y \\
= & \frac{2}{\Delta z} \int_{1}^{\infty} \frac{d y}{y} \int_{0}^{\frac{\Delta z}{2}} \epsilon^{-y z} d z=\frac{2}{\Delta z} \int_{1}^{\infty}\left(\frac{1}{y^{2}}-\frac{e^{-y \Delta z}}{y^{2}}\right) d y \\
= & \frac{2}{\Delta z}\left(1-\epsilon^{-\frac{\Delta z}{2}}\right)+E_{1}\left(\frac{\Delta z}{2}\right)
\end{aligned}
$$

The integrating scheme is the equivalent of the trapezoidal rule but employing the function of mean argument instead of the chordal mean as the surmation elenent.

One proceeds by inserting a tabular trial function, performing the matrix operation as indicated and emergine which is iterated until the variation produced by iteration is insignificant. 

In practice minor departures from the straight forward process were introduced to reduce the labor. The principal one of these consisted in iterating for only about 10 terms of the flux vector and writing the complete vector by smoothing the first differences.

A variant of this scheme was considered, which involved writing the equation in a perturbation form:

$$
\varphi_{a(z)}-\delta_{(z)}=\frac{\Sigma_{t}}{2 \Sigma} \int_{-\infty}^{\infty}\left[E_{1\left(\left|z^{\prime}-z\right|\right)}-\varepsilon_{\left(\left|z^{\prime}-z\right|\right)}\right]\left[\varphi_{a(z)}-\delta_{(z)}\right] d z
$$

where $\varphi_{a}$ is the asymptotic solution, $\delta$ is the boundary depression, and $\varepsilon$ is the perturbation of the operator $E_{1}$ required to extend the integration over all $z$.

$$
\delta(z)=\frac{\Sigma_{t}}{2 \Sigma} \int_{-\infty}^{\infty}\left\{\left[E_{1\left(\left|z^{\prime}-z\right|\right)}-\varepsilon_{\left(\left|z^{\prime}-z\right|\right)}\right] \delta(z)+\varepsilon \varphi_{a}\right\} d z
$$

or in Riemann sum form

$$
\delta_{i}=\frac{\Sigma_{t}}{2 \Sigma} \Delta z \sum\left[E_{i}-\varepsilon\right]_{\left(\left|z_{i}-z_{n}\right|\right)} \delta_{n}+\sum_{n} \varepsilon_{\left(\left|z_{i}-z_{n}\right|\right)} \varphi_{a}
$$

The equivalent matrices, all infinite order are:

$\varphi_{a}=\left[\begin{array}{c}- \\ \bar{\varphi} \\ \varphi_{-2} \\ \varphi_{-1} \\ \varphi_{1} \\ \varphi_{2} \\ - \\ \overline{-}\end{array}\right] \quad \delta=\left[\begin{array}{c}- \\ \overline{-} \\ \varphi_{-2} \\ \varphi_{-1} \\ \delta_{1} \\ \delta_{2} \\ - \\ -\end{array}\right] \quad \varepsilon=\left[\begin{array}{cccccc}- & - & - & - & - & - \\ - & - & - & - & - & - \\ - & E_{-2-2} & E_{-2-1} & E_{-21} & E_{-22} & - \\ - & E_{-1-2} & E_{-1-1} & E_{-11} & E_{-12} & - \\ - & E_{1-2} & E_{1-1} & 0 & 0 & 0 \\ - & E_{2-2} & E_{2-1} & 0 & 0 & 0 \\ - & - & - & 0 & 0 & 0\end{array}\right]$ 


$$
E-\varepsilon=\left[\begin{array}{llllllll}
0 & 0 & 0 & 0 & 0 & 0 & 0 & 0 \\
0 & 0 & 0 & 0 & 0 & 0 & 0 & 0 \\
0 & 0 & 0 & 0 & 0 & 0 & 0 & 0 \\
0 & 0 & 0 & 0 & 0 & 0 & 0 & 0 \\
0 & 0 & 0 & E_{11} & E_{12} & E_{13} & - & - \\
0 & 0 & 0 & E_{21} & E_{22} & E_{23}- & - \\
0 & 0 & 0 & E_{31} & E_{32} & E_{33} & - \\
0 & 0 & 0 & - & - & - & - \\
0 & 0 & 0 & - & - & - & - \\
& & & & & &
\end{array}\right]
$$

The first method has the disadvantage that the term products converge rather slowly for increasing $z$ so that each term of the iterated vector represents very many term products and the computation is quite laborious. The second method avoids this trouble since $\delta$ vanishes strongly with increasing $z$, but $\delta$ varies so rapidly near $(z=0)$ that trapezoidal integration is valid only for a very small interval $\Delta z$. On balance, method one appeared most feasible and was chosen. A value of $2.9 \mathrm{~cm}^{-1}$ was obtained for $\Sigma$ with a very fine fit of the solution to the experimental data.

To investigate the validity of the integration, the process was then repeated with an improved kernel. In this case the matrix elements were obtained by computing mean values for the exponential integral with a unit weight function for each $\Delta \boldsymbol{z}_{n}$. This process amounts to correcting for variation of the kernel over the interval but ignoring the variation of the function. A value of $\Sigma=2.5 \mathrm{~cm}^{-1}$ resulted.

A further improvernent in the kernel was then tried. One assumes that the function ray be linearized with small error over the interval $\Delta z$. 
The matrix equation is then written:

$$
\varphi_{\left(z_{i}\right)}=\frac{\Sigma_{t}}{2 \Sigma} \sum \int_{n}\left[\varphi_{\left(z_{n}\right)}+\frac{\Delta \varphi_{n}}{\Delta z} \xi\right] E_{1}\left(\left|z_{i}-z_{n}+\xi\right|\right) d \xi
$$

where $z_{n}$ is the lower boundary of the $n^{\text {th }}$ interval, $\Delta z$ is the width of the interval, $\Delta \varphi_{n}$ is the difference $\left[\varphi\left(z_{n+1}\right)-\varphi\left(z_{n}\right)\right]$, and $\zeta$ is the difference $\left[z-z_{n}\right]$. The equation may be separated into two terms as follows:

$$
\begin{aligned}
\varphi_{\left(z_{i}\right)}=\frac{\Sigma_{t}}{2 \Sigma} \sum_{n}\left[\varphi_{\left(z_{n}\right)} \int_{0}^{\Delta z} E_{1}\left(\left|z_{i}-z_{n}+\xi\right|\right) d \xi\right. \\
\left.+\frac{\Delta \varphi_{n}}{\Delta z} \int_{0}^{\Delta z} \rho E_{1}\left(\left|z_{i}-z_{n}+\xi\right|\right) d \xi\right]
\end{aligned}
$$

The first integral (letting $y=\left|z_{i}-z_{n}+\xi\right|=\left|K_{n}+\xi\right|$ )

$I_{1, n}=\int_{K_{n}}^{K_{n+1}} E_{1(y)} d y=e^{-K_{n}}-e^{-K_{n+1}}-K_{n} E_{1}\left(K_{n}\right)+K_{n+1} E_{1}\left(K_{n+1}\right)$

The second integral, dropping absolute values:

$$
\begin{aligned}
& I_{2, n}=\int_{K_{n}}^{K_{n+1}}\left(y-K_{n}\right) E_{1}(y) d y \\
& =\frac{1}{2}\left[e^{-K_{n}\left(1+K_{n}\right)-e^{-K_{n+1}}\left(1+K_{n+1}\right)+K_{n+1}^{2} E_{1}\left(K_{n+1}\right)}-K_{n}^{2} E_{1}\left(K_{n}\right)\right]-K_{n} I_{1, n}
\end{aligned}
$$

$I_{1}$ constitutes the terms of an operator which operates on the function $\varphi_{n}$ and $I_{2}$ an operator which operates on the relative increment of the function, $\Delta \varphi_{n} / \Delta z$ 
The process yielded again an excellent fit to the data but for a value $\Sigma=2.3 \mathrm{~cm}^{-1}$. Since the computing had become impractically tedious, further work on this line was dropped.

Frankel and Goldberg (3) list a solution for the boundary flux as follows :

$$
\varphi_{(0)}=\sqrt{\frac{1}{2}\left(\frac{\sum_{t}}{\sum\left[1-d^{2}\right]}-1\right)}
$$

where the asymptotic flux goes as the hyperbolic sine vanishing at an "extrapolated" boundary thus:

$$
\varphi_{a}(z)=\sinh x\left(z+z_{0}\right)
$$

The extrapolation distance is given by:

$$
Z_{0}=\frac{1}{\pi} \int_{0}^{1} \frac{1}{1-\alpha^{2} s^{2}} \tan ^{-1}\left(\frac{\pi / 2}{\tanh ^{-1} s-\Sigma / s \Sigma_{t}}\right) d s
$$

The characteristic equation of the system relates $\mathcal{H}$ to $\frac{\Sigma_{t}}{\Sigma}$, so the boundary flux may be expressed as a function of $\mathbb{R}$ alone:

$$
\begin{aligned}
& 2 \varphi_{(0)}^{2}=\frac{\Sigma_{t}}{\Sigma\left(1-x^{2}\right)}-1 \\
& \frac{\Sigma}{\Sigma_{t}}\left(1-x^{2}\right)=\frac{1}{1+2 \varphi_{(0)}^{2}} \\
& \left(1-x^{2}\right)\left(1+\frac{x^{2}}{3}+\frac{x^{4}}{5}+\cdots\right)=\frac{1}{1+2 \varphi_{(0)}^{2}} \\
& \frac{2 x^{2}}{3}+\frac{2 x^{4}}{15}+\frac{2 x^{6}}{35}+\cdots \frac{2 x^{2 n}}{(2 n+1)(2 n-1)}=1-\frac{1}{1+2 \varphi_{(0)}^{2}} \\
& x^{2}+\frac{x^{4}}{5}+\frac{x^{6}}{7}+\ldots \frac{x^{2 n}}{2 n+1}=\frac{3 \varphi_{(0)}^{2}}{1+2 \varphi_{(0)}^{2}} \\
& 20
\end{aligned}
$$


The procedure is as follows:

1. The extrapolation distance is computed by Simpson's Rule for a few values of $\mathcal{X}$ in the neighborhood of $0.15-0.20$ as indicated by the numerical solution, and plotted against $\mathcal{X}$.

2. Selecting a value of $\mathcal{H}$ and the corresponding $Z_{0}$, the experimental boundary flux is normalized to go as $\sinh \mathscr{H}\left(z+z_{0}\right)$ at large argument. This new nornalization is obtained by halving that flux which was nornalized to go asymptotically as $\epsilon^{\mathscr{H} z}$ and multiplying by $e^{d z_{0}}$ The result is $\varphi_{(0)}$.

3. Insert the $\varphi_{(0)}$ in the series equation and solve by trial for $\mathfrak{R}$.

4. Using the computed values of $\mathcal{H}$, return to stage (2) and iterate the process. Convergence is rapid.

The described process yielded $\Sigma=2.10 \pm 0.05 \mathrm{~cm}^{-1}$. 
CHAPTER III

\section{EXPER TIENTAL PROCEDURE}

The medium in which the experiment was conducted was the approximately $6^{\prime} \times 6^{\prime} \times 6^{\prime}$ water tank which is mounted on the thermal column of the Oak Ridge Graphite Reactor. A current of thermal neutrons passes upward through the tank after filtering through the graphite column below. The water content is ion-exchange demineralized, and its nuclear properties are substantially those of distilled water.

A disk of aluminum, faced with .020 inch cadmium, and approximately 10" in diameter, carried on aluminum legs about 10" long, was lowered with a rod to a reproducible central location on the tank bottom. A foil holder of aluminum and lucite, fastened to the cadmium face was provided with adjustable mountings for positioning foils along the central normal to the plane. A foil was also cemented to the center of the cadmium plane for each exposure. The foil mountings were of lucite of minimum section consistent with stiffness, and a film of paraffin was employed as an adhesive to mount the foils, thus introducing no non-hydrogenous material in the immediate vicinity of the foils. The equipment is shown as Fig. (3).

Indium foils were used, weighing 25 milli-grams nominal, cut from $50 \mathrm{mg} / \mathrm{cm}^{2}$ stock. These were cleaned of the adhesive wax after each use, weighed and allowed to decay for at least 48 hours before re-use.

Foil counting was done with an end mica-window Geiger-Mueller counter, connected through a plate-loaded pre-amplifier stage (of gain $\sim 12$ ) to a scale of 64. Counter and pre-amplifier were enclosed in a $2^{\text {" }}$ lead shield, which reduced background to a negligible fraction of the foil activity. Sufficient counts were obtained in each counting run to reduce statistical 
fluctuation to a negligible level. A number of determinations of counter resolving time were made by following the decay of $54 \mathrm{~min}$ indium 116 over about 5 hours. An average value of $240 \mu$ sec. was obtained by solving the following:

$$
\frac{A_{1}}{1-N_{1} \tau}=\frac{A_{2}}{1-N_{2} \tau}
$$

where $A=$ extrapolated activity

$\mathrm{N}=$ mean counting rate, $\mathrm{sec}^{-1}$

$\tau=$ resolving time, sec.

A correction factor $1 / 1-N \sigma$ was plotted against counting rate and applied to all counting runs to correct for coincidence losses.

For exposure, the foil holders (after mounting the foils) were adjusted approximately to position with respect to the cadmium plane using a common scale. A vernier height gauge was then used to determine the exact foil position (estimated probable error $\pm .05 \mathrm{~mm}$.). The plane and foil holder assembly was then lowered quickly to the tank bottom and exposed for times ranging from 5 to 15 minutes depending on foil position to obtain reasonable counting rates. No attempt was made to measure either exposure or foil "cooling" times accurately since one foil always and two foils usually were exposed at fixed positions to monitor the activity of the variable foil. The positions at $x=0$ and $x \cong 5.18 \mathrm{~cm}$. are the monitor positions and data at these points represents an average of about 15 measurements. In addition to the exposures made with axial foils, two exposures were made with foils set in a plane parallel to the cadmium to demonstrate that the geometry was actually one dimensional. A plot of the diametral variation of flux appears as Fig. (6). The maximur deviation from the axial flux amounts to about 
$5 \%$ reduction at $8 \mathrm{~cm}$. radius. This is considered satisfactory. To test the flux for fast components, a cadmium difference on Indium was taken. The ratio of activity in Cd. to activity in the "shadow" of the cadmium envelope (a most conservative condition) exceeded 5,000 - therefore foil activation due to the 1.44 volt resonance may be safely neglected.

To analyze the data by the numerical method, it was first plotted against the coordinate $x$ in cms on semi-log paper and the fair curve drawn by eye. A clearly exponential branch was obtained beginning at $x \sim 4.8 \mathrm{~cm}$. The decay constant $\mathrm{k}$ of the exponential was solved by

$$
k=\frac{\ln \frac{\varphi(a)}{\varphi(b)}}{a-b}
$$

and fixed at $k=0.375$. The scale of the values of $\varphi(x)$ was then adjusted so that $\varphi$ (asymptotic) $=e^{0.375 x}$. A trial value of $\Sigma$ was then selected, and the data plotted, faired and tabulated as a function of $z$ in mean path measure. The eigenvalue $\frac{\Sigma_{t}}{2 \Sigma}$ was computed, the $E_{1}(|m \Delta z|)$ tabulated on a strip for $\Delta z=.2$, and numerical iteration of the equation carried out until it was obvious that the solution near the plane at $z=0$ would not fit the data. The trial was then terminated and another trial made for a different value of $\Sigma$.

As the solutions were improved it became clear that a correction was necessary for the remainder contributed by the terms beyond the last tabular value of the $E_{1}$. The correction was inserted by computing the remainder with respect to a proper exponential function and dividing by the value of this function at the first untabulated term of the $E_{1}$. This number is then used to terminate the $\mathrm{E}_{1}$ tabulation. Since the last tabulated value of the $E_{1}$ was for $m \Delta z=7.4$ one obtains: 


$$
e^{-7.6 x} \int_{7.5}^{\infty} e^{x z} E_{1(z)} d z
$$

which may be integrated by writing the $E_{1}$ as an integral, then inverting the order of integration to yield

$$
\frac{1}{\not} \epsilon^{-.18}\left\{-E_{1}(7.5)+\frac{1}{d x} \varepsilon^{-7.5 x} E_{1}(7.5-7.5 \not)\right\}
$$

which must be computed individually for each value of $\Sigma$.

Iteration was carried out by computing only every fifth term of the trial function, then interpolating the new function by making the first differences regular. The final integration was done in detail in the region close to the black boundary.

Plots of the flux as a function of distance from the black boundary appear as Fig。 (4) and (5). The curves were obtained by using the Le Caine results to interpolate the data near the boundary, and the numerically iterated function in the region more than $0.5 \mathrm{~cm}$. from the boundary. The latter process is considered reasonable since the solutions in this region were invariant to changes in the integrating scheme, they are constrained by the approach to the asymptotic function, and they fit the data quite well. Desirable extensions to the analysis might include:

(1) Computation of the Wiener-Hopf solution for perhaps 15 or 20 points to firm up confidence in the fit.

(2) Extensive analysis of the numerical iteration method to establish the level of computing de il necessary to yield accurate solutions using the true values of the parameters. Both propositions require computing machine service to reduce program time to a practical value. 


\section{UNCLASSIFIED \\ OWG. 10140}

FIG. I

VELOCITY DIAGRAM FOR TWO BODY COLLISION

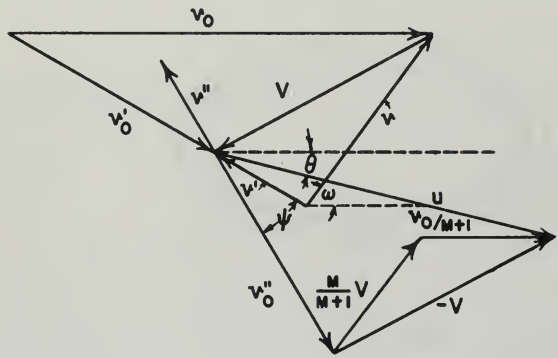


$x^{2}$

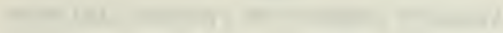

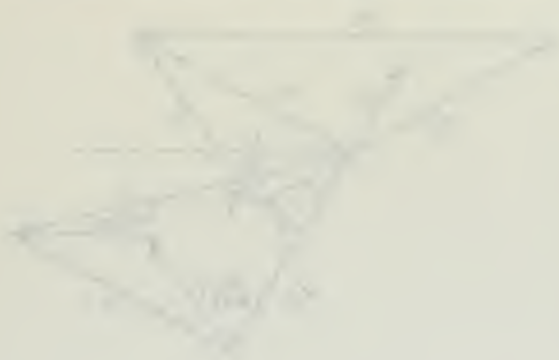


UNCLASSIFIED

DWG. 10141

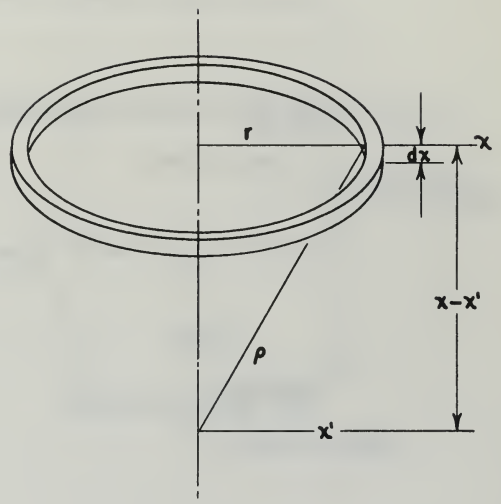

FIG. 2

INTEGRATION FOR PLANE KERNEL - TRANSPORT EQUATION 


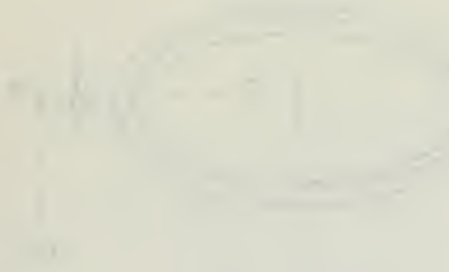


UNCLASSIFIED

DWG. 10144

FIG. 3

FOIL MOUNT AND CADMIUM PLANE ASSEMBLY
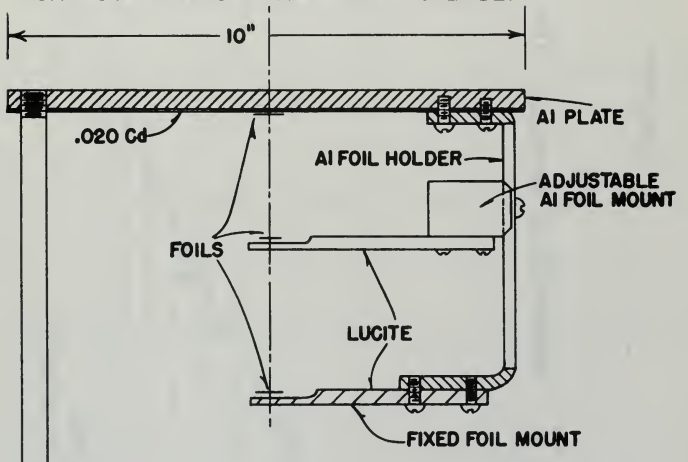

AI LEG - I OF 3

Not to scale 


\section{$x^{-x^{-4}}=$}

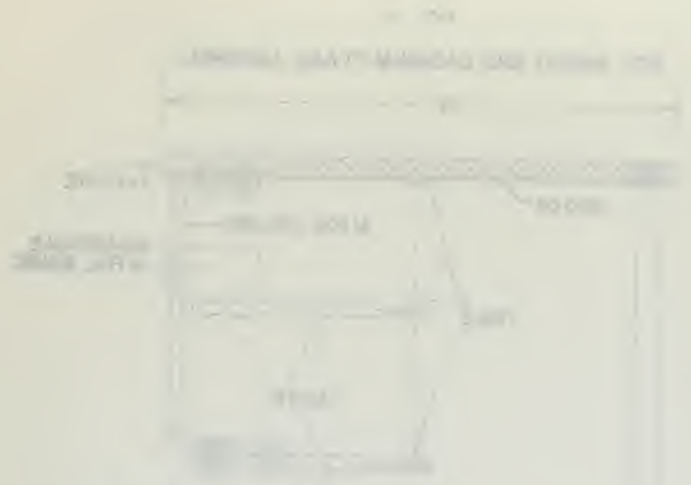

int :

s.

new 


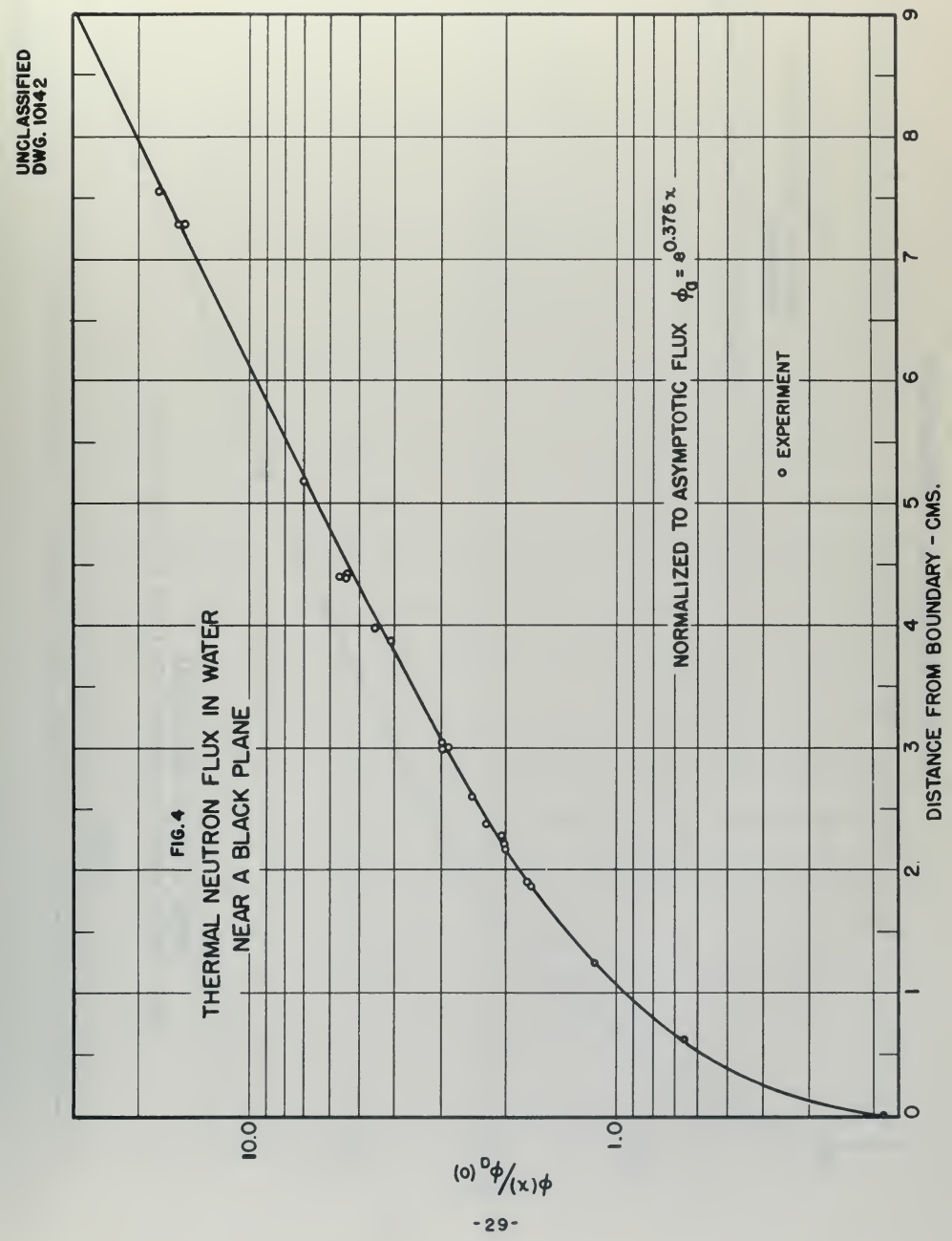




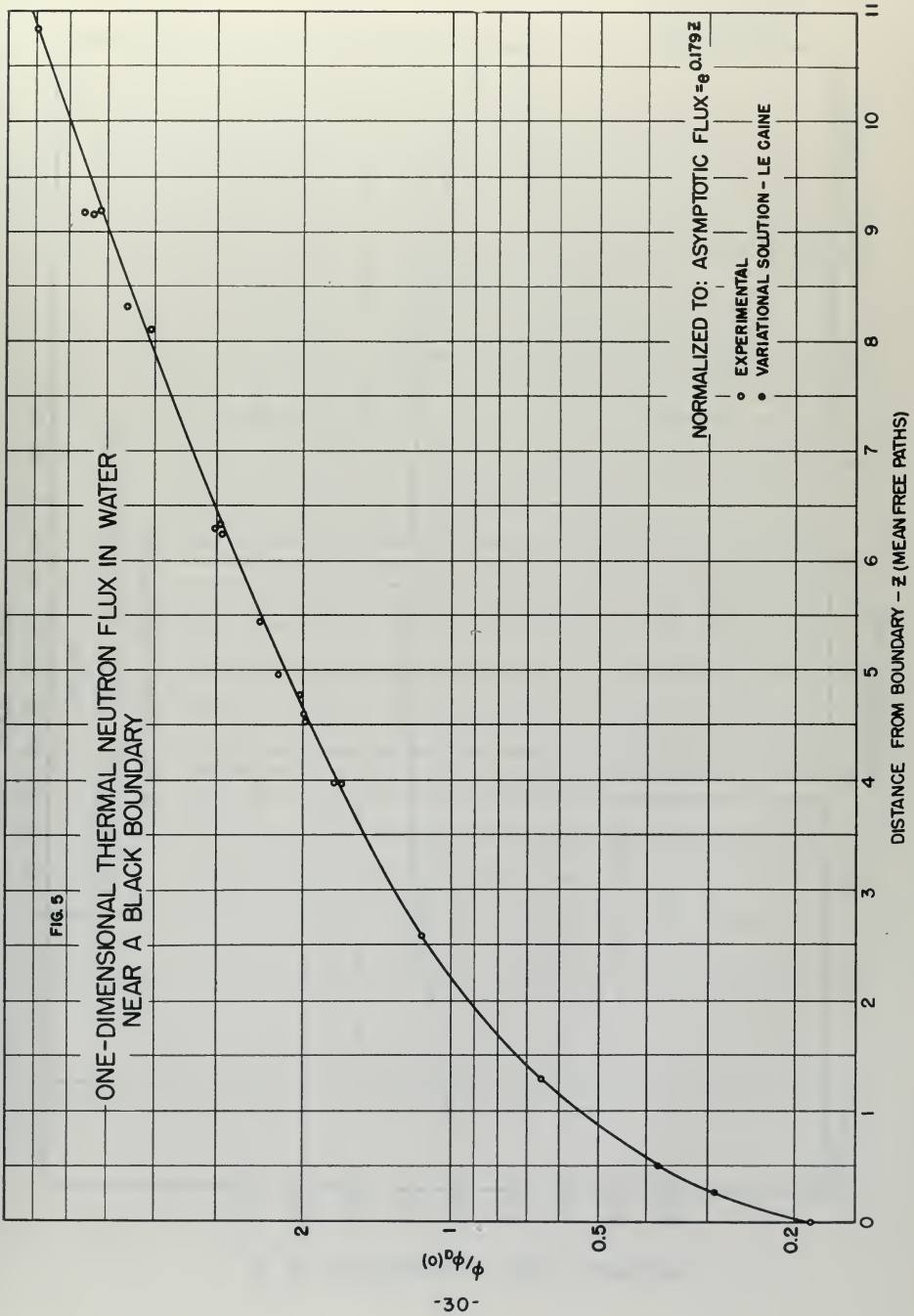





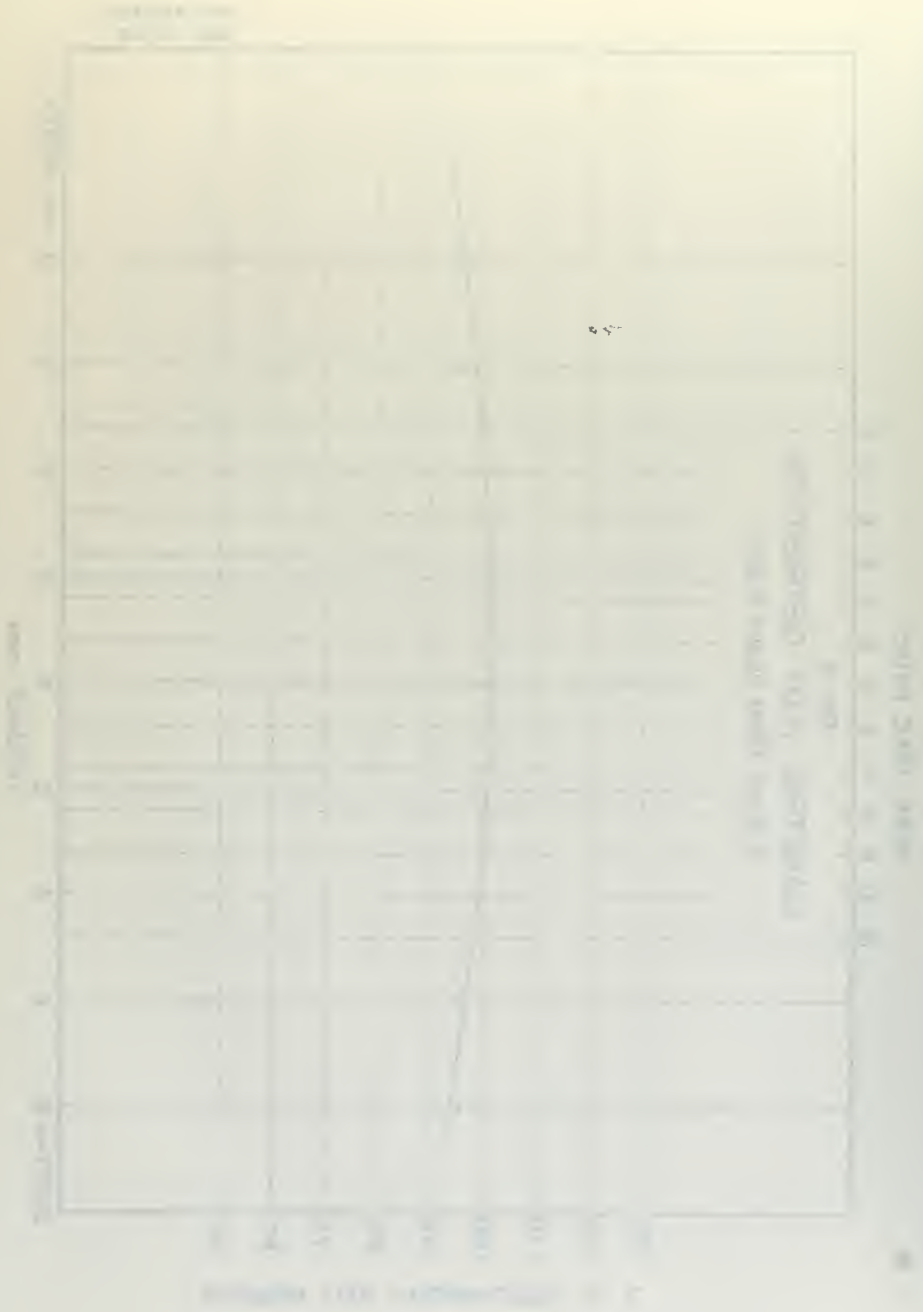




\section{B IBLIOGRAPHY}

1. *The Slow Neutron Cross Section of Nater as a Function of Energy. Bacher et al. CP-1146 (Declassified)

2. *iNeutron Physics, J. G. Beckerley (A Revision of Halpern's notes on E. Fermi's 1945 Lectures) AECD 2664.

3. *The Wathematical Development of the End-Point Wethod.

S. Frankel \& Goldberg

4. *A Method of Treatment of Displacenent Integral Equations. Frankel \& Nelson, LA-56 (Secret).

5. On the Scattering of Thermal Neutrons. F'. Joliot. Contes Rendues Vol. 228 p. 245-6.

6. Neutron Flux Near a Black Plane. J. LeCaine, Canadian Journal of Research 28-3A p. 242 .

7. *Slow Neutron Velocity Spectrometer Studies. E. Lielkmian. AECD-2675 (Unclassified).

8. On the Decrease of Neutron Density at a Boundary. Raether, H. Zeitschriff fur Physic. Vol. $125 \mathrm{p} .269-273$.

9. Vibrational Spectra and Structure of Polyatomic Molecules, Ta-You Wu Publ. J. W. Edwards, Ann Arbor, 1946.

*AEC Publications. 
APrENDIX I

EXPERIMENTAL IAIA -

COMPUTATION OF ACTIVITIES 
Foil Inventory

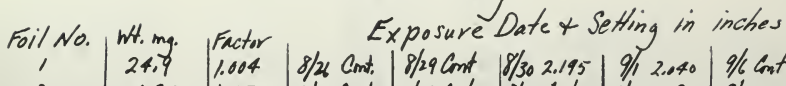

4

5

6

7

8

9

10

"I

12

13

14 \begin{tabular}{l|l}
24.35 & 1.027
\end{tabular} $24.3 \quad 1.029$

$23.5 \quad 1.064$ $25.2 \quad 0.992$

23.7 $24.9 \quad 1.004$ $22.4 \quad \mathrm{H} / 1 \mathrm{6}$ 24,5 23.2 22.9 1.020 1.078 1.092 $23.35 \quad 1.071$ 24.9 $23.7 \quad 1.055$ $6 / 13$ Cont $8 / 26$ Cont. $8 / 29$ Cont 8/302.195 $6 / 130.490 \quad 8 / 262.026 \quad 8 / 29$ Cont. \begin{tabular}{ll|llll}
$6 / 13$ & 2.043 & $8 / 26$ & 0.866 & $8 / 29$ & 1.192
\end{tabular} $6 / 13$ Cont. 8/26 Cont 8/24 2.043 $6 / 130.798$ d/26 0.866 8/50 Cont. L/13 2.043 8/26 $2.043 \mathrm{8} / 29$ Cnt $8 / 210.2448 / 296025 \quad \% / 12.040$ $8 / 272043 \quad \% / 292.040$ $8 / 27$ Cont $8 / 300.854$ $8 \% 302.036 \quad 8 \% 312.036$ 9)1 2.030 9/2 Cont. \%) Cont. a/s 2.030 q/1 $2.038 \quad 9 / 22.030$ r/30 2.195 $8 / 302.195$ $8 / 302.195$ $9 / 12.040$ $9 / 12.040$ $9 / 12.040$ $8 / 31$ Cnt $8 / 311.567$ g) 1.721 $9 / 61.524$ $9 / 10.748$ $9 / 62.023$ $9 / 81.185$ of cont. q/6 Cont

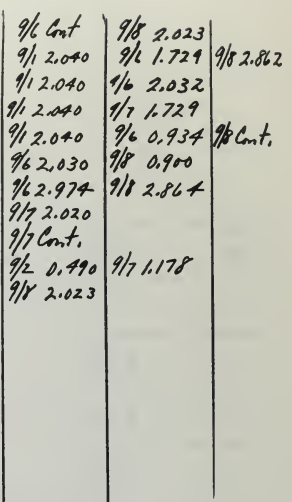



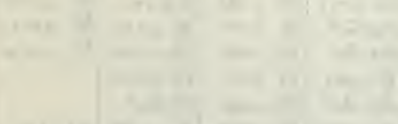

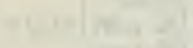
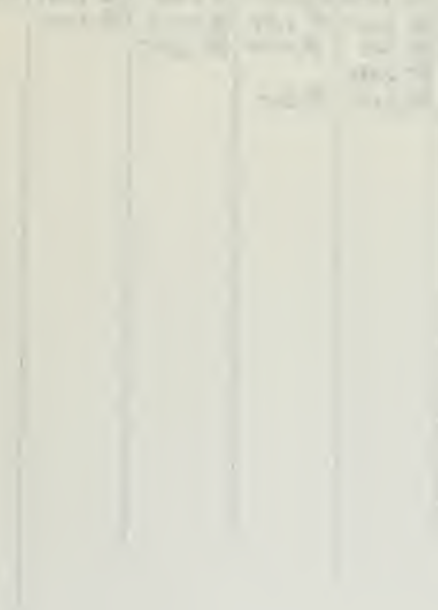
了是是焉

h $\quad \sigma^{2}$

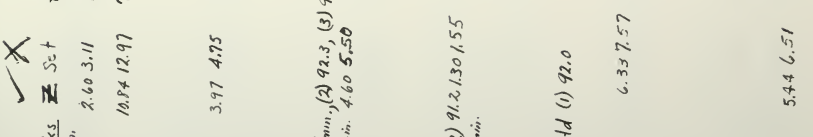

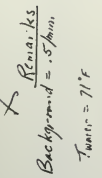

कू⿻

ส?

हा

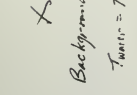

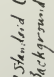

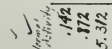

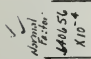

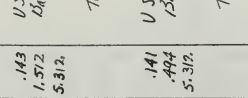

के

กू้

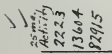

रहत

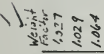

จิ้ พู้

骨

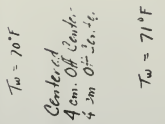

$\frac{k}{2}$

13

स

के

$\operatorname{i⿻n} \frac{m}{n} d i$

1)

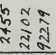

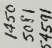

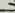

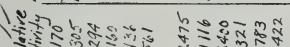

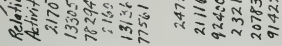

ริ?

药

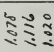

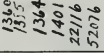

ฝูปูล

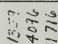

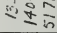

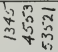

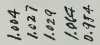

ริำำ

Ā

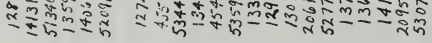

हैंड़े

के

हैं के के

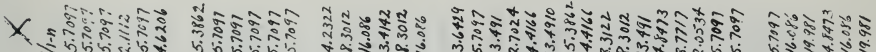

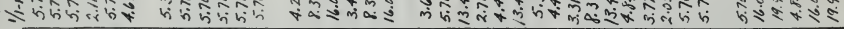
Xะ1

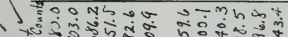

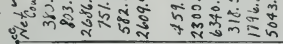

गुำ

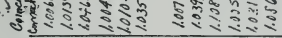
1)

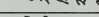

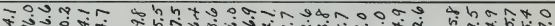

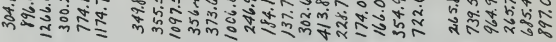

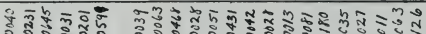
क्षेत्र

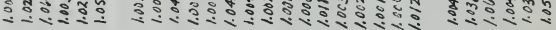

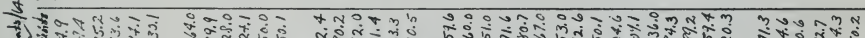

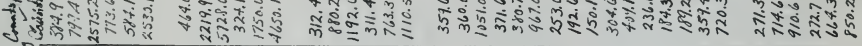
गी

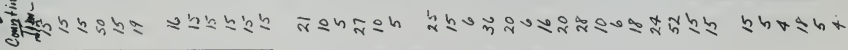

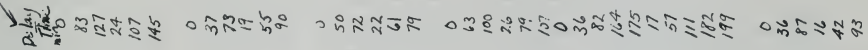

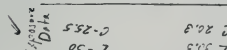

$\sin 3$

$01-2$

$21-9$

$21-3$

11-3

$5 /-3$

80

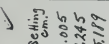

กิ

น: : ह

ถิ่ พิ\%

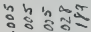

में

क सेक्ष

ऊ

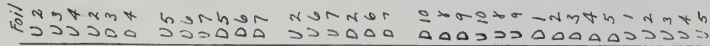

$N \infty \omega+\infty$ 


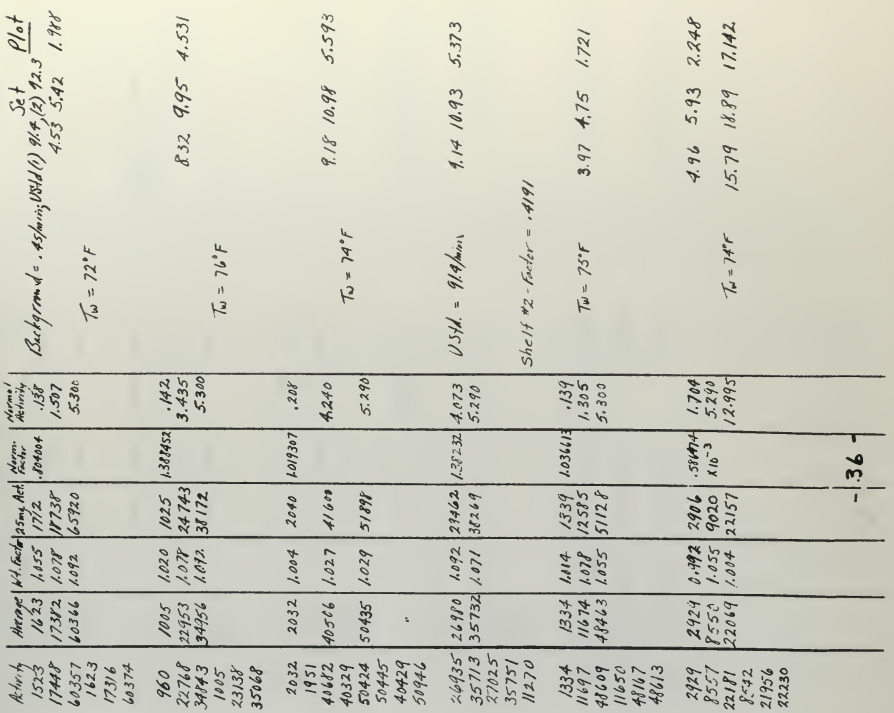

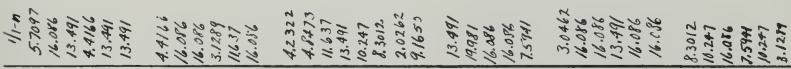

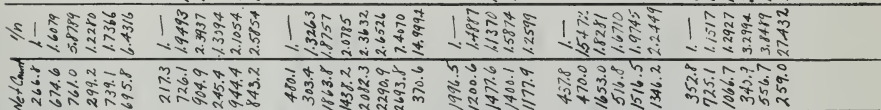

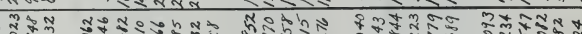

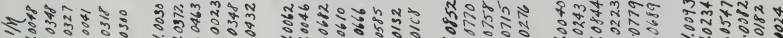

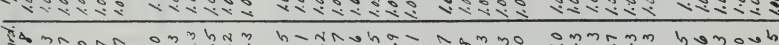

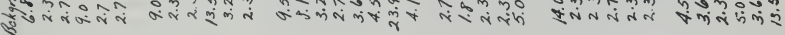

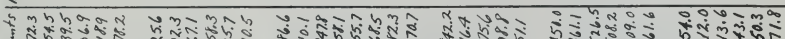
न

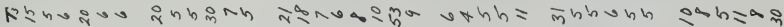

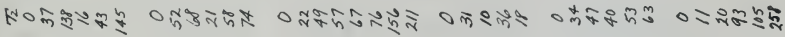

\begin{tabular}{|c|c|c|c|c|c|c|}
\hline $\begin{array}{c}21-7 \\
6-9 \\
4\end{array}$ & $\begin{array}{l}61-0 \\
11-9\end{array}$ & $\begin{array}{r}\varepsilon-2 \\
51-3\end{array}$ & & $\begin{array}{l}5 z-3 \\
z 1-7\end{array}$ & $\begin{array}{l}5-2 \\
2 x-3\end{array}$ & $\begin{array}{c}3<-0 \\
5.7\end{array}$ \\
\hline 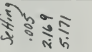 & $\frac{h}{2}$ & फ & है & रुजै & ถิฐะิ & 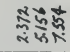 \\
\hline
\end{tabular}

iัง

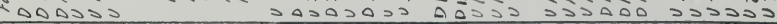





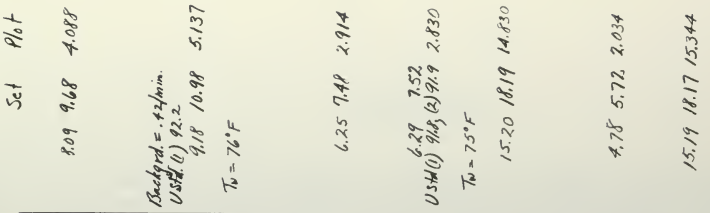

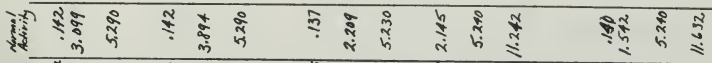

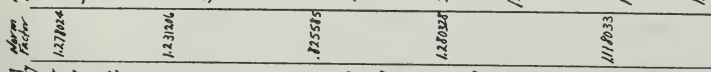

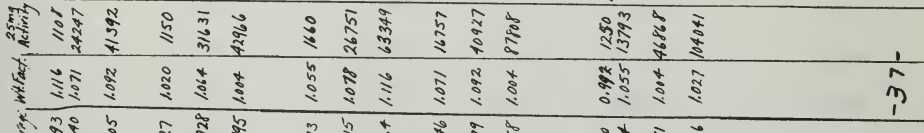

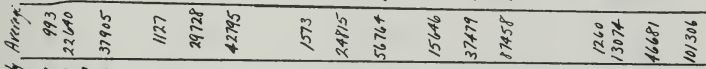

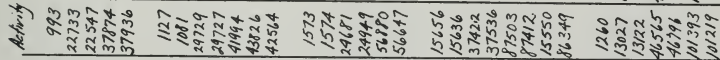

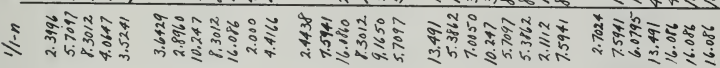

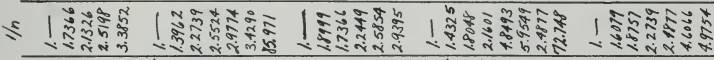

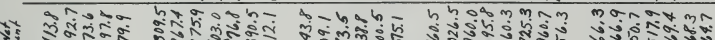

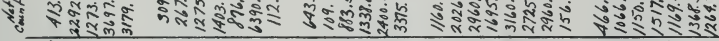

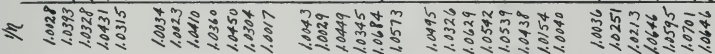

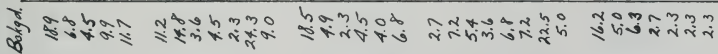

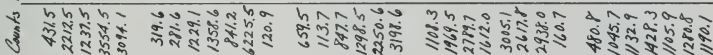

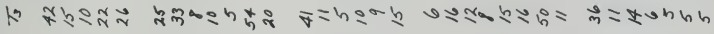

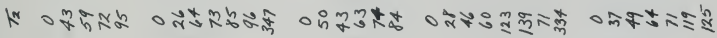

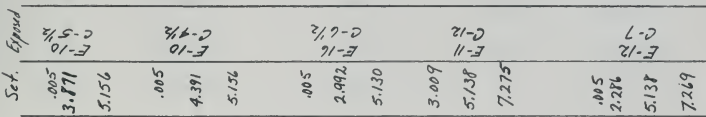

ए人

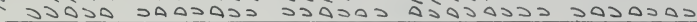

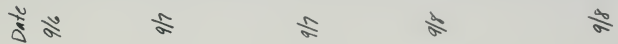


The flux is tabulated as a function of $Z$ for two values of the total cross section. These are solutions corresponding (for $\Sigma=2.3$ ) to the most elaborate numerical treatment, and (for $\Sigma=2.1$ ) to the best reconciliation of Wiener-Hopf, variational, and numerical and experimental solutions.

\begin{tabular}{|c|c|c|c|c|c|}
\hline$z$ & $\phi(\Sigma=2.1)$ & $\phi(\Sigma=2.3)$ & $z$ & $\phi(\Sigma=2.1)$ & $\phi(\Sigma=2.3)$ \\
\hline 0 & 1.86 & 1.86 & 4.2 & 18.00 & 16.45 \\
\hline 0.2 & 2.71 & 2.70 & 4.4 & 18.90 & 17.20 \\
\hline 0.4 & 3.44 & 3.45 & 4.6 & 19.80 & 18.00 \\
\hline 0.6 & 4.15 & 4.14 & 4.8 & 20.75 & 18.80 \\
\hline 0.8 & 4.84 & 4.80 & 5.0 & 21.75 & 19.70 \\
\hline 1.0 & 5.52 & 5.44 & 5.2 & 22.75 & 20.60 \\
\hline 1.2 & 6.20 & 6.07 & 5.4 & 23.80 & 21.50 \\
\hline 1.4 & 6.88 & 6.69 & 5.6 & 24.90 & 22.40 \\
\hline 1.6 & 7.57 & 7.30 & 5.8 & 26.00 & 23.25 \\
\hline 1.8 & 8.29 & 7.93 & 6.0 & 27.10 & 24.20 \\
\hline 2.0 & 9.04 & 8.59 & 6.2 & 28.20 & 25.20 \\
\hline 2.2 & 9.84 & 9.25 & 6.4 & 29.50 & 26.20 \\
\hline 2.4 & 10.64 & 9.93 & 6.6 & 30.70 & 27.25 \\
\hline 2.6 & 11.49 & 10.60 & 6.8 & 32.00 & 28.30 \\
\hline 2.8 & 12.30 & 11.30 & 7.0 & 33.40 & 29.45 \\
\hline 3.0 & 13.05 & 12.00 & 7.2 & 34.80 & 30.55 \\
\hline 3.2 & 13.85 & 12.75 & 7.4 & 36.10 & 31.75 \\
\hline 3.4 & 14.65 & 13.50 & 7.6 & 37.60 & 33.00 \\
\hline 3.6 & 15.45 & 14.25 & 7.8 & 39.10 & 34.20 \\
\hline 3.8 & 16.30 & 14.95 & 8.0 & 40.80 & 35.50 \\
\hline 4.0 & 17.15 & 15.70 & & & \\
\hline
\end{tabular}



WS. Mo thi

3) 7 



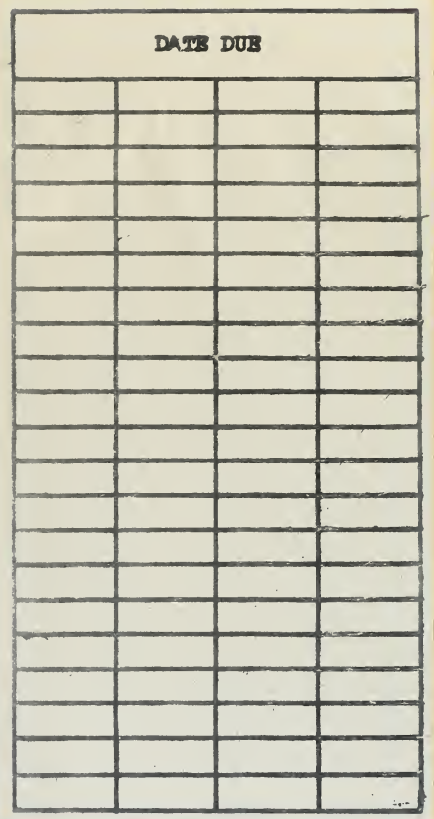




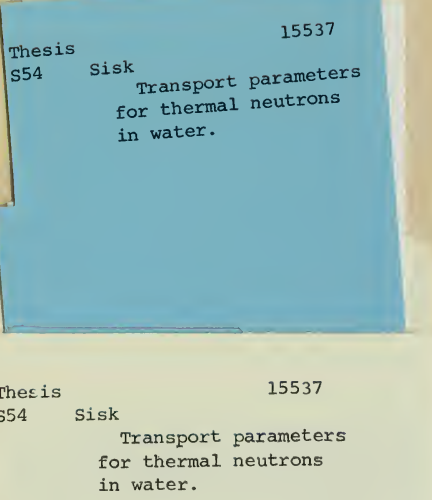


